Bull. Korean Math. Soc. 45 (2008), No. 4, pp. 651-661

\title{
THE SEMIGROUPS OF BINARY SYSTEMS AND SOME PERSPECTIVES
}

\author{
Hee Sik Kim And Joseph Neggers
}

\begin{abstract}
Given binary operations "*" and "०" on a set $X$, define a product binary operation " $\square$ " as follows: $x \square y:=(x * y) \circ(y * x)$. This in turn yields a binary operation on $\operatorname{Bin}(X)$, the set of groupoids defined on $X$ turning it into a semigroup $(\operatorname{Bin}(X), \square)$ with identity $(x * y=x)$ the left zero semigroup and an analog of negative one in the right zero semigroup $(x * y=y)$. The composition $\square$ is a generalization of the composition of functions, modelled here as leftoids $(x * y=f(x))$, permitting one to study the dynamics of binary systems as well as a variety of other perspectives also of interest.
\end{abstract}

$\S$ 0. In what follows we associate with a set $X$ a $\operatorname{semigroup}(\operatorname{Bin}(X)$, $\square)$ which generalizes in a rather natural way the composition of functions $f$ : $X \rightarrow X$. Classes of groupoids defined on a set $X$ may form semigroups or even ideals and the axioms defining these classes may be analyzed according to the properties of these classes in $(\operatorname{Bin}(X), \square)$ for the set $X$. The fact that some sets of axioms produce subsemigroups of $(\operatorname{Bin}(X), \square)$ for the set $X$, whereas other sets do not, appears to be an interesting and possibly important distinction for the class under discussion. Some classes which appear to be new are also pointed out, including the linear groupoids over rings with identity, usually taken to be commutative. The strong bounded $d$-algebras are shown to be an interesting class described as a convenient intersection of three other classes each determined by one of the axioms. Given the standard "equivalence" these algebras will (indirectly) satisfy several $M V$-axioms. Other properties such as separability are looked at as well.

$\S$ 1. Given a set $X$ and a function $f: X \rightarrow X$ consider a groupoid $(X, *, f)$ where the multiplication is given by the formula

$$
x * y=f(x) .
$$

If $f(x)=i d_{X}(x)=x$, then $\left(X, *, i d_{X}\right)$ has a multiplication

$$
x * y=x
$$

Received June 26, 2007.

2000 Mathematics Subject Classification. 20N02.

Key words and phrases. leftoid, semigroup, binary system, orientation (property), (travel, linear) groupoid, orbit, strong, $d$-algebra, separable. 
and the groupoid is referred to as the left zero semigroup. In keeping with this terminology we shall refer to groupoids of the type $(X, *, f)$ as leftoids.

Consider leftoids $(X, *, f)$ and $(X, \circ, g)$. Define an operation " $\square$ " on these leftoids as follows:

$$
(X, *, f) \square(X, \circ, g)=(X, \square)
$$

where

$$
x \square y=(x * y) \circ(y * x) .
$$

Thus it follows that $x \square y=f(x) \circ f(y)=g f(x)$, i.e., $(X, \square)$ is the leftoid $(X, \square, f \circ g)$, i.e., the multiplication as in (4) corresponds to the composition of functions from $X$ to $X$. Since composition of functions is an associative operation, we obtain the following:

Theorem 1. The collection of leftoids with respect to the operation $\square$ is a semigroup with identity $\left(X, *, i d_{X}\right)$.

$\S$ 2. Suppose now that for a given set $X$ we consider $\operatorname{Bin}(X)$, the collection of all binary systems (groupoids, algebras) defined on $X$. Given arbitrary groupoids $(X, *)$ and $(X, \circ)$, define a product $(X, \square)=(X, *) \square(X, \circ)$ precisely as in formula (4). Then we have the following:

Theorem 2. $(\operatorname{Bin}(X), \square)$ is a semigroup, i.e., the operation $\square$ as defined in general is associative. Furthermore, the left zero semigroup is an identity for this operation.

Proof. If $((X, *) \square(X, \circ)) \square(X, \nabla)=(X, \triangle)$, then a typical product is

$$
x \triangle y=((x * y) \circ(y * x)) \nabla((y * x) \circ(x * y)) .
$$

Similarly, $(X, *) \square((X, \circ) \square(X, \nabla)$ has a typical element $(x * y) \sim(y * x)$, where $a \sim b=(a \circ b) \nabla(b \circ a)$, i.e., it is $((x * y) \circ(y * x)) \nabla((y * x) \circ(x * y))=x \triangle y$ and the associativity follows.

Also $(X, *) \square\left(X ; \circ, i d_{X}\right)=(X, \nabla)$, where $x \nabla y=(x * y) \circ(y * x)=x * y$ and $\left(X ; \circ, i d_{X}\right) \square(X, *)$ has a typical element $(x * y) \circ(y * x)=x * y$. Hence $(X, \nabla)=(X, *)$ in either case.

$\S$ 3. Suppose that in $\operatorname{Bin}(X)$ we consider all those groupoids $(X, *)$ with the orientation property: $x * y \in\{x, y\}$ for all $x$ and $y$. Thus, $x * x=x$ as a consequence. If $(X, *)$ and $(X, \circ)$ both have the orientation property, then for $x \square y=(x * y) \circ(y * x)$ we have the possibilities: $x * x=x, y * y=y, x * y \in\{x, y\}$ and $y * x \in\{x, y\}$, so that $x \square y \in\{x, y\}$. It follows that if $O P(X)$ denotes this collection of groupoids, then $(O P(X), \square)$ is a subsemigroup of $(\operatorname{Bin}(X)$, $\square)$. The left zero semigroup has $x * y=x \in\{x, y\}$, i.e., it is an element of $O P(X)$. We summarize:

Proposition 3. $(O P(X), \square)$ is a semigroup with identity. 
A different interpretation of $O P(X)$ is the following: For $(X, *) \in O P(X)$, if $x \neq y$ and $x * y=y$ draw an arrow from $x$ to $y$, and if $x * y=x$ do not draw such an arrow. The resulting picture is a digraph (without loops at the vertices) and conversely any such digraph generates a groupoid $(X, *)$ in $O P(X)$.

From our observation above we have defined an associative product of digraphs with vertex set $X$.

In a sequence of papers Nebeský $[6,7,8]$ has sought to associate with graphs $(V, E)$ groupoids $(V, *)$ with various properties and conversely. Although not identical in outlook there is some similarity. In particular, Nebeský defines a travel groupoid $(X, *)$ as a groupoid satisfying the axioms: $(u * v) * u=u$ and $(u * v) * v=u$ implies $u=v$. If one adds these two laws to the orientation property, then $(X, *)$ is an OP-travel-groupoid. In this case $u * v=v$ implies $v * u=u$, i.e., $u v \in E$ implies $v u \in E$, i.e., the digraph $(X, E)$ is an (simple) graph if $u u \notin E$, with $u * u=u$. Also, if $u \neq v$, then $u * v=u$ implies $(u * v) * v=u * v=u$ is impossible, whence $u * v=v$ and $u v \in E$, so that $(X, E)$ is a complete (simple) graph. On the other hand if $(X, E)$ is a complete (simple) graph, then $u \neq v$ implies $u v \in E$ and $u * v=v$, whence $(X, *)$ is the right zero semigroup in any case. Given that $(u * v) * v=v$ as a consequence, it follows that $(u * v) * v=u$ implies $u=v$ and $(X, *)$ is an OP-travel-groupoid.

$\S$ 4. Let $(R,+, \cdot, 0,1)$ be a commutative ring with identity and let $L(R)$ denote the collection of groupoids $(R, *)$ such that for all $x, y \in R$

$$
x * y=a x+b y+c,
$$

where $a, b, c \in R$ are fixed constants. We shall consider such groupoids to be linear groupoids. Notice that $a=1, b=c=0$ yields $x * y=1 \cdot x=x$, and thus the left zero semigroup on $R$ is a linear groupoid.

Now, suppose that $(R, *)$ and $(R, \circ)$ are linear groupoids where $x * y=$ $a x+b y+c$ and $x \circ y=d x+e y+f$. Then $x \square y=d(a x+b y+c)+e(a y+b x=$ $c)+f=(d a+e b) x+(d b+e a) y+(d+e) c+f$, whence $(R, \square)=(R, *) \square(R, \circ)$ is also a linear groupoid. We summarize:

Proposition 4. $(L(R), \square)$ is a semigroup with identity.

Many common operations are of the linear type. Thus $a=b=1, c=0$ yields $x * y=x+y$. Also $a=1, b=-1, c=0$ yields $x * y=x-y$. In this case $x \square y=(x+y)-(y+x)=0$ and $x \square y=(x-y)+(y-x)=0$ produces a different view of what we mean by the statement: "plus and minus are inverse operations". The linear groupoids over the real numbers $\mathbb{R}$ form a very large class of groupoids with many different types among them.

$\S 5$. Given a groupoid $(X, *)$ we define the orbit $\Omega(X, *)$ to consist of groupoids $(X, *(n))$, where $(X, *(1))=(X, *)$ and $(X, *(n+1))=(X, *(n)) \square$ $(X, *(1))$. Since $(\operatorname{Bin}(X), \square)$ is a semigroup it follows that if $i+j=k$, then $(X, *(i)) \square(X, *(j))=(X, *(k))$ as well. 
It follows immediately that $(\Omega(X, *), \square)$ is the principal semigroup generated by the algebra $(X, *)$ and that the fact that it is a semigroup allows one to discuss the orbital dynamics in much the same way that one does for iterated function systems for example. In terms of the physical importance of this fact, one should note that it may well be the case that in complicated situations it may not be the best way to describe systems via functions or operators but through groupoids/algebras instead. To our knowledge this is the first observation noting that it is possible.

To construct the basin of attraction $B A(X, *)$ we proceed as follows:

(1) $B A_{1}(X, *)=\Omega(X, *)$;

(2) If $B A_{n}(X, *)$ has been defined and if $\Omega(X, \circ) \cap B A_{n}(X, *) \neq \emptyset$, then $(X, \circ) \in B A_{n+1}(X, *)$;

(3) Since $(X, *) \in \Omega(X, *)$, it follows that $(X, \circ) \in B A_{n}(X, *)$ implies $\Omega(X, \circ) \cap B A_{n}(X, *) \neq \emptyset$ and thus $B A_{n}(X, *) \subseteq B A_{n+1}(X, *) ;$

(4) Let $B A(X, *)=\cup_{n \in \omega} B A_{n}(X, *)=\lim _{n \rightarrow \infty} B A_{n}(X, *)$.

For example, if $\mathbb{R}$ is the real numbers and $(\mathbb{R}, *)=(\mathbb{R},+)$, then $x+2(n) y=$ $(x+y)+(y+x)=2(x+y)$ and $x+(n+1) y=2^{n}(x+y)$. Notice that $\Omega(\mathbb{R},+)$ is non-periodic. The presence of "exponential growth" is quite evident in this particular example

Suppose that $|(x+y)-(a+b)|=\rho(x+y, a+b)>0$ for $(\mathbb{R},+)$. Then we find that $\mid(x+(n+1) y)-\left(a+(n+1) b \mid=2^{n} \rho(x+y, a+b)\right.$ so that for $\Omega(\mathbb{R},+)$ we also note that there is "sensitive dependence on initial conditions."

Given a real number $\alpha$, if $x_{n}+y_{n}=2^{-n+1} \alpha$, then also $x_{n}+(n) y_{n}=$ $2^{n-1}\left(x_{n}+y_{n}\right)=2^{n-1}\left(2^{-n+1} \alpha\right)=\alpha$, so that the sequence $\left\{x_{1}+y_{1}, x_{2}+\right.$ $\left.y_{2}, \ldots, x_{n}+y_{n}, \ldots\right\}$ has the property that it shows up "in the neighborhood of $\alpha$ " for the operations $x+(n) y, n=1,2, \ldots$

Usually the three properties we have illustrated above when taken together mean that the orbit, in this case $(\mathbb{R},+)$, is chaotic. Precise rules can be provided in the general situation certainly.

If we consider an operation on $\mathbb{R}, x \oplus y=\frac{1}{\sqrt{2}}(x+y)$, then $x \oplus y$ is of the linear type and $x \square y=(x \oplus y) \oplus(y \oplus x)=\frac{1}{\sqrt{2}}\left(\frac{1}{\sqrt{2}}(x+y)+\frac{1}{\sqrt{2}}(y+x)\right)=x+y$, so that $(\mathbb{R}, \oplus) \in B A_{2}(\mathbb{R},+)$ and $(\mathbb{R}, \oplus) \in B A_{1}(\mathbb{R},+)$. If we set $x \odot y=-\frac{1}{\sqrt{2}}(x+y)$, then $x \square y=(x \odot y) \odot(y \odot x)=x+y$ as well. These appear to be the only linear groupoids in $B A_{2}(\mathbb{R},+)-B A_{1}(\mathbb{R},+)$, "directly below" $(\mathbb{R},+)$, i.e., $(\mathbb{R}, \oplus) \square(\mathbb{R}, \oplus)=(\mathbb{R}, \odot) \square(\mathbb{R}, \odot)=(\mathbb{R},+)$.

Generally, if $x * y=\frac{2^{n}}{\sqrt{2}}(x+y)$, then $x \square y=(x * y) *(y * x)=\frac{2^{n}}{\sqrt{2}}\left(\frac{2^{n}}{\sqrt{2}}(x+\right.$ $\left.y)+\frac{2^{n}}{\sqrt{2}}(y+x)\right)=\frac{2^{2 n+1}}{\sqrt{2}} \sqrt{2}(x+y)=2^{2 n}(x+y)$ and $(\mathbb{R}, \square) \in \Omega(\mathbb{R},+)$ whereas $(\mathbb{R}, *)=\left(\mathbb{R},+\left(n-\frac{1}{2}\right)\right) \notin \Omega(\mathbb{R},+)$ produces other elements of $B A_{2}(\mathbb{R},+)-$ 
$B A_{1}(\mathbb{R},+)$. All these are linear groupoids. It appears that there may not be non-linear groupoids in $B A(\mathbb{R},+)$.

If $(\mathbb{R}, *)$ has $x * y=\frac{1}{2}(x+y)$, i.e., $a=b=\frac{1}{2}, c=0$, then $(x * y) *(y * x)=$ $\frac{1}{2}\left(\frac{1}{2}(x+y)+\frac{1}{2}(y+x)\right)=\frac{1}{2}(x+y)=x * y$, and thus $(\mathbb{R}, *)=(\mathbb{R}, *(2))$, so that also $(\mathbb{R}, *)=(\mathbb{R}, *(n))$ for all $n$, whence $\Omega(\mathbb{R}, *)=\{(\mathbb{R}, *)\}$. If $a(a x+b y+c)+$ $b(a y+b x+c)+c=\frac{1}{2}(x+y)$, then $\left(a^{2}+b^{2}\right) x+2 a b y+(a+b+1) c=\frac{1}{2}(x+y)$ yields $(a-b)^{2}=0$, so that $a=b=-\frac{1}{2}$. Since $a^{2}+b^{2}=-\frac{1}{2}$ is impossible in $\mathbb{R}$ there is no linear groupoid $(R, \circ)$ such that $(R, \circ) \square(R, \circ)=(R, *)$.

$\S$ 6. We let $O P^{\prime}(X)$ consist of those $(X, *)$ such that $x * y=y$ implies $y * x=y$. In terms of the associated digraphs this means that $x \rightarrow y$ means $y \nrightarrow x$ and the digraph is an orientation. Orientations are important in the theory of digraphs. Suppose $(X, *)$ and $(X, \circ)$ are in $O P^{\prime}(X)$. Suppose also that $x \square y=(x * y) *(y * x)=y$. If $x * y=x$, then $y * x=y$ and $x \square y=x * y=y$, an impossibility. Hence $x * y=y=y * x$ so that $y \square x=x$ for all $x, y \in X$. Therefore it is an element of $O P(X)$. We summarize:

Proposition 5. $O P^{\prime}(X)$ is a subsemigroup of $(O P(X), \square)$ having an identity.

If $(X, *) \in O P^{\prime}(X)$ and $x \square y=(x * y) *(y * x), x * y=x$ yields $x \square y=$ $x *(y * x)=x$ since $x * y=x * x=x, x * y=y$ yields $x \square y=(x * y) *(y * x)=$ $y * y=y$. Thus, $x \square y=x * y$ in any case, i.e., $(X, *(2))=(X, *)$ whence $\Omega(X, *)=\{(X, *)\}$, which proves the following:

Proposition 6. All orbits of $O P^{\prime}(X)$ are singletons.

Notice that if $(X, *) \in O P(X)$ and if $x \square y=(x * y) *(y * x)=y$, then $x * y=x$ implies $x *(x * y)=y$ whence $x * y=y$, an impossibility. Thus $x * y=y$ and $y *(y * x)=y$. If $y * x=x$, then $(y * x) *(x * y)=x * y=y=y \square x=x \square y$. If $y * x=y$, then $(y * x) *(x * y)=y * y=y=y \square x=x \square y$. We summarize:

Proposition 7. If $(X, *) \in O P(X)$, then $(X, *(2)) \in O P^{\prime}(X)$.

If $(X, *) \in O P(X)-O P^{\prime}(X)$, then for some $x, y \in X, x \neq y, x * y=y$ and $y * x=x$. Consider $(X, *(3))$. We have

$$
\begin{aligned}
x *(3) y & =((x * y) *(y * x)) *((y * x) *(x * y)) \\
& =(y * x) *(x * y) \\
& =x * y \\
& =y
\end{aligned}
$$

and $x *(2) y=(x * y) *(y * x)=y * x=x$. Hence $(X, *(3))=(X, *)$ and $(X, *(2)) \neq(X, *(1))$ so that $\Omega(X, *)=\{(X, *),(X, *(2)),(X, *(3))=$ $(X, *), \ldots\}$, i.e., it has precisely two elements.

Given a poset $(X, \leq)$, we let $x * x=x$ and if $x<y$, then $x * y=y, x * y=x$ otherwise. Hence, if $x<y$, then $x * y=y$ and $y * x=y$, i.e., $(X, \leq)$ generates an 
element $(X, *)$ of $O P^{\prime}(X)$ with the additional property that $x * y=y, y * z=z$ implies $x * z=z$, when $x \neq y, y \neq z$.

$\S$ 7. Given $(X, *) \in \operatorname{Bin}(X)$, we shall consider it strong if $x * y=y * x$ implies $x=y$. If $(X, *)$ and $(X, \circ)$ are strong, then $x \square y=(x * y) \circ(y * x)=$ $(y * x) \circ(x * y)=y \square x$ implies $x * y=y * x$ and hence $x=y$ as well. We summarize:

Proposition 8. If $\operatorname{Str}(X)$ consists of all strong groupoids, then $(\operatorname{Str}(X), \square)$ is a semigroup with identity.

Given $\operatorname{Bin}(X)$, let $Z_{a}(X)$ consist of all $(X, *)$ such that $x * x=a$ for all $x \in X$. Then, if $(X, *)$ and $(X, \circ)$ are in $Z_{a}(X)$, we have $(X, *) \square(X, \circ)=(X, \square)$ with $x \square x=(x * x) \circ(x * x)=a \circ a=a$, so that $(X, \square) \in Z_{a}(X)$, i.e., $Z_{a}(X)$ is a semigroup. In this case the left zero semigroup is not an element of $Z_{a}(X)$. In addition note that $Z_{a}(X) \square Z_{b}(X) \subseteq Z_{b}(X)$. In fact $\operatorname{Bin}(X) \square Z_{a}(X) \subseteq Z_{a}(X)$. Indeed, $(x * x) \circ(x * x)=a=x \square x$ in that case. Also, $(x \circ x) *(x \circ x)=a * a=x \square x$ which varies with $(X, *) \in \operatorname{Bin}(X)$. Hence $\left(Z_{a}(X), \square\right)$ is a left ideal of $(\operatorname{Bin}(X)$, $\square)$. We summarize:

Proposition 9. $\left(Z_{a}(X), \square\right)$ is a left ideal of $(\operatorname{Bin}(X), \square)$.

Given $\operatorname{Bin}(X)$ let $N_{a}(X)$ consist of all $(X, *)$ such that $a * x=a$ for all $x \in X$. If $(X, *)$ and $(X, \circ)$ are elements of $N_{a}(X)$, then $a \square x=(a * x) \circ(x * a)=$ $a \circ(x * a)=a$ and $\left(N_{a}(X), \square\right)$ is a semigroup with identity, since the left zero semigroup belongs to $N_{a}(X)$ for all $a \in X$. In fact, if $(X, *) \in \cap_{a \in X} N_{a}(X)$, then $(X, *)$ is the left zero semigroup precisely. We summarize:

Proposition 10. $\left(N_{a}(X), \square\right)$ is a semigroup with identity.

It follows that $\left(\operatorname{Str}(X) \cap Z_{a}(X) \cap N_{a}(X), \square\right)$ is a semigroup. We note that $(X, *, 0)$ is a d-algebra [11] provided (i) $x * x=0$; (ii) $0 * x=0$; (iii) $x * y=$ $y * x=0$ if and only if $x=y$. If (iii) $x * y=y * x$ if and only if $x=y$, then we consider the $d$-algebra to be strong. Otherwise we consider the $d$-algebra to be exceptional. For details, see [9, 10]

The strong $d$-algebras are those $(X, *)$ which belong to $\operatorname{Str}(X) \cap Z_{0}(X) \cap$ $N_{0}(X)$, which automatically determine a semigroup by the observation made above. Strong $d$-algebras have been discussed in [3] as a subclass of the class of $d$-algebras with particular properties of interest both by themselves as well as in their relationship with the larger class of $d$-algebras.

Example 11. Given a poset $(X, \leq)$ with minimal element 0 , the standard $B C K$-algebra $[4,5]$ for this poset is defined as $(X, *, 0)$ where $x * y=0$ if $x \leq y, x * y=x$ otherwise. Thus $x * x=0,0 * x=0$. Assume that $x * y=y * x$. If $x \leq y$, then $y * x=x * y=$, i.e., $y \leq x$. Since $(X, \leq)$ is a poset, $x=y$. Similarly it holds for the case $y \leq x$ also. Otherwise, $x * y=x=y * x=y$. Hence $(X ; *, 0)$ is a strong $d$-algebra. 
Example 12. Given a field $K$, let $(K, *)$ be the groupoid where $x * y=x(x-y)$. Then $x * x=0,0 * x=0$ and $x * y=y * x=0$ implies $x=y$. On the other hand, $x * y=y * x$ means $x(x-y)=y(y-x)$ or $(x+y)(x-y)=0$ and $x=y$ or $x=-y$. Hence if $\operatorname{char}(K) \neq 2$, then $(K, *, 0)$ is an exceptional $d$-algebra.

$\S$ 8. Suppose $(X, *)$ contains elements 0 and $b$ such that $0 * x=0$ and $x * b=0$ for all $x \in X$. If $b_{1}$ and $b_{2}$ are type $b$ elements with respect to 0 , then $b_{1} * b_{2}=b_{2} * b_{1}=0$. Hence, if $(X, *, 0)$ is a $d$-algebra, then $b_{1}=b_{2}$ and $b$ is unique. Under these circumstances if $(X, *)$ is of this type, then $x \square b=(x * b) \circ(b * x)=0 \circ(b * x)=0$ if $(X, \circ)$ has the same 0 , while $0 \square x=(0 * x) \circ(x * 0)=0 \circ(x * 0)=0$ as well if this is the case. Thus, if $(X, \circ) \in N_{0}(X)$ and $(X, *) \in N_{0}^{b}(X)$, where $N_{0}^{b}(X)$ consists of all $(X, *, 0, b)$ with $0 * x=0$ and $x * b=0$, then $N_{0}^{b}(X) \square N_{0}(X) \subseteq N_{0}^{b}(X)$.

Combining this with the result on strong $d$-algebras we observe that "for bounded strong $d$-algebras the bound is unique and the product $(X, *, 0, b)$ $\square(X, *, 0)$ of strong $d$-algebras is again a bounded strong $d$-algebra. It follows that the semigroup of bounded strong $d$-algebras is a right ideal in the semigroup of strong $d$-algebras (sharing the 0 element).

Using the notation $\neg x=b * x, x \oplus y=((\neg x) *((\neg x) *(\neg y)))$ we can then seek to add further conditions on $(X, *)$ so as to obtain $M V$-algebras $(X, \oplus)$ (see $[1,2])$ with some added properties perhaps.

$\S$ 9. Suppose that $A b(X)$ consists of all groupoids $(X, *)$ which are abelian (commutative), i.e., $x * y=y * x$ for all $x, y \in X$. Note that if $(X, *) \in A b(X)$ and $(X, \circ)$ is an arbitrary groupoid, then in $(X, *) \square(X, \circ), x \square y=(x * y) \circ(y * x)=$ $(y * x) \circ(x * y)=y \square x$. Also, $(X, \circ) \square(X, *)=(X, \square)$ with $x \square y=(x \circ y) *(y \circ x)=$ $(y \circ x) *(x \circ y)=y \square x$. We summarize:

Proposition 13. $(A b(X), \square)$ is a two-sided ideal of $(\operatorname{Bin}(X), \square)$

This indicates that commutativity is a rather stronger property than others which do not generate two-sided ideals. Of course, if a two-sided ideal $(I, \square)$ contains the left zero semigroup then $I=\operatorname{Bin}(X)$. Hence properties which are satisfied by the left zero semigroup can only be measured in terms of ideals (types) by considering their complements.

Suppose for example that $(X, *)$ is not strong. Then for some $x \neq y, x * y=$ $y * x$. Hence, if $(X, \circ)$ is arbitrary, then $(X, *) \square(X, \circ)$ has $x \square y=(x * y) \circ(y * x)=$ $(y * x) \circ(x * y)=y \square x$, where $x \neq y$. We summarize:

Proposition 14. $(\operatorname{Bin}(X)-\operatorname{Str}(X), \square)$ is a right ideal of $(\operatorname{Bin}(X), \square)$.

Combining Propositions 8 and 14 yields an interesting decomposition of $(\operatorname{Bin}(X), \square)$ into the union of a right ideal and a subsemigroup with identity analogous to that of a local ring with identity into a group of units and a maximal ideal. 
Theorem 15. If $(X, \circ)$ has the property that $X \circ X=X$, and given $x, y$, there are $a, b$ such that $a \circ b=x, b \circ a=y$, then $(X, \circ) \square(X, *)$ is also in $(\operatorname{Bin}(X)-\operatorname{Str}(X), \square)$.

Proof. Let $x * y=y * x, x \neq y$. Then $a \circ b=x, b \circ a=y$ implies $a \neq b$. Furthermore $a \square b=(a \circ b) *(b \circ a)=x * y=y * x=(b \circ a) *(a \circ b)=b \square a$, so that $(X, \square)$ is not strong, as asserted.

If $(X, *)$ has the property that $\Omega(X, *) \cap A b(X) \neq \emptyset$, then $(X, *(n)) \in A b(X)$ implies $(X, *(n+1))=(X, *(n)) *(X, *) \in A b(X)$ as well. Thus, if $H A b(X)$ consists of all $(X, *)$ such that $\Omega(X, *) \cap A b(X) \neq \emptyset$, then we may associate with $(X, *)$ a hypo-abelian index $n$ where $n$ is the smallest positive integer such that $(X, *(n)) \in A b(X)$, with $n=1$ meaning $(X, *(1))=(X, *)$ is abelian.

Example 16. If $(G, \cdot, e)$ is a group, then we shall consider it "hypo-abelian" if $x y^{2} x=y x^{2} y$ for all $x, y \in G$, i.e., $x \square y=(x \cdot y) \cdot(y \cdot x)=(y \cdot x) \cdot(x \cdot y)=y \square x$ in terms of our language developed here. There are many non-abelian groups of this type, e.g., the dihedral group $D_{4}$ and the quaternion group $Q$ both of order 8 are of this type.

Example 17. Let $(\mathbb{R}, *)$ be defined by $x * y=\frac{1}{2} x+y$. Then $2 * 1=2$ and $1 * 2=\frac{5}{2}$, and $(\mathbb{R}, *) \notin A b(\mathbb{R})$. Now, $(\mathbb{R}, *(2))$ has $x *(2) y=(x * y) *(y * x)=$ $\frac{1}{2}\left(\frac{1}{2} x+y\right)+\left(\frac{1}{2} y+x\right)=\frac{5}{4} x+\frac{3}{2} y, x *(3) y=(x *(2) y) *(y *(2) x)=\frac{1}{2}\left(\frac{5}{4} x+\frac{3}{2} y\right)+$ $\left(\frac{5}{4} x+\frac{3}{2} y\right)=\frac{17}{8} x+2 y$, etc.. With a little work one shows $\Omega(\mathbb{R}, *) \cap A b(X)=\emptyset$ and thus $(\mathbb{R}, *) \notin H A b(\mathbb{R})$.

$\S$ 10. Given a function $\mu: X \rightarrow \mathbb{R}$, let $\mu \operatorname{Dom}(X)$ consists of all $(X, *)$ such that $\mu(x * y) \geq \min \{\mu(x), \mu(y)\}$. If $\operatorname{Im} \mu \subseteq[0,1]$, then $(X, *)$ is a fuzzy subalgebra. If $(X, *)$ and $(X, \circ)$ are elements of $\mu \operatorname{Dom}(X)$, then $(X, *) \square(X, \circ)$ yields $\mu(x \square y)=\mu((x * y) \circ(y * x)) \geq \min \{\mu(x * y), \mu(y * x)\} \geq \min \{\mu(x), \mu(y)\}$. We summarize:

Proposition 18. $(\mu \operatorname{Dom}(X), \square)$ is a subsemigroup of $(\operatorname{Bin}(X), \square)$ with identity.

Indeed, $x * y=x$ implies $\mu(x * y)=\mu(x) \geq \min \{\mu(x), \mu(y)\}$.

If we let $\mu \operatorname{Dom}^{*}(X)$ consist of all $(X, *)$ such that $\mu(x * y) \leq \max \{\mu(x), \mu(y)\}$, then $(X, *) \square(X, \circ)$ yields $\mu(x \square y)=\mu((x * y) \circ(y * x)) \leq \max \{\mu(x * y), \mu(y * x)\} \leq$ $\max \{\mu(x), \mu(y)\}$. We summarize:

Proposition 19. $\left(\mu \operatorname{Dom}^{*}(X), \square\right)$ is a subsemigroup of $(\operatorname{Bin}(X), \square)$.

If one is interesting in fuzzy aspects of the theory of groupoids, these propositions may make good starting points for further development.

$\S$ 11. Given $(X, *) \in \operatorname{Bin}(X)$, let $F(X, *)=\{\mu: X \rightarrow \mathbb{R} \mid(X, *) \in$ $\mu \operatorname{Dom}(X)\}$. Then $\lambda>0$ implies $\lambda \mu(x * y) \geq \min \{\lambda \mu(x), \lambda \mu(y)\}$. Also, 
$\mu, \nu \in F(X, *)$ implies $\mu+\nu \in F(X, *)$, so that $F(X, *)$ is a cone. Similarly, $F^{*}(X, *)=\left\{\mu: X \rightarrow \mathbb{R} \mid(X, *) \in \mu \operatorname{Dom}^{*}(X)\right\}$ is a cone. Hence, if $(X, *)$ is the left zero semigroup, then $F(X, *)=F^{*}(X, *)=\mathbb{R}^{X}$.

Since $(X, *) \in \mu \operatorname{Dom}(X)$ implies $(X, *(n)) \in \mu \operatorname{Dom}(X)$ and thus $F(X, *(n))$ $\supseteq F(X, *)$, with $F^{*}(X, *(n)) \supseteq F^{*}(X, *)$ one may impose an "order" on $\operatorname{Bin}(X)$ by setting $(X, *) \leq(X, \circ)$ provided $F(X, *) \subseteq F(X, \circ)$ with maximal elements including the left zero semigroup for example.

Questions involve comparisons of groupoids $(X, *)$ and $(X, \circ)$ for which $F(X, *)=F(X, \circ), F^{*}(X, *)=F^{*}(X, \circ)$, etc..

For example, if $(X, *)$ has $x * y \notin\{x, y\}$, let $\mu(x)=\mu(y)=1$ and $\mu(z)=0$ if $z \notin\{x, y\}$, then $\mu(x * y)=0<\min \{\mu(x), \mu(y)\}$ and thus $(X, *) \notin \mu \operatorname{Dom}(X)$. Hence, if $F(X, *)=\mathbb{R}^{X}$, then $x * y \in\{x, y\}$. On the other hand, if $x * y \in\{x, y\}$, then $\mu(x * y) \geq \min \{\mu(x), \mu(y)\}$ for all $\mu \in \mathbb{R}^{X}$.

$\S$ 12. As a final perspective, suppose $(X, \cdot)$ is an abelian groupoid, then $(X, *)$ is said to be separable over $(X, \cdot)$ if $x * y=f(x) \cdot g(y)$ for some maps $f, g: X \rightarrow X$. If $g(y)=a$ is a constant, then $x * y=f(x) \cdot g(y)=f(x) a$ implies certain leftoids are separable over $(X, \cdot)$. If $x \cdot e=x$ for all $x \in X$, then $g(y)=e$ and $x * y=f(x)=f(x) \cdot e$ implies all leftoids are separable over $(X, \cdot)$.

If we consider $x \square y=(x * y) \cdot(y * x)=f(x * y) \cdot g(y * x)=f(f(x) \cdot g(y))$. $g(f(y) \cdot g(x))$. In general we may not expect that $(X, \square)$ is separable over $(X, \cdot)$.

If $\varphi:(X, \cdot) \rightarrow(X, \cdot)$ is an endomorphism, then $\varphi(x \cdot y)=\varphi(x) \cdot \varphi(y)$ and thus if we define $x * y=\varphi(x) \cdot \varphi(y)=\varphi(x \cdot y)$, then $(X, *)$ is separable over $(X, \cdot)$. Now $(x * y) \cdot(y * x)=\varphi(x * y) \cdot \varphi(y * x)=\varphi(\varphi(x) \cdot \varphi(y)) \cdot \varphi(\varphi(y) \cdot \varphi(x))=$ $\varphi^{2}(x) \cdot \varphi^{2}(y) \varphi^{2}(y) \cdot \varphi^{2}(x)=\left(\varphi^{2}(x) \cdot \varphi^{2}(x)\right) \cdot\left(\varphi^{2}(y) \cdot \varphi^{2}(y)\right)$ and thus $(X, *(2))$ is again separable over $(X, \cdot)$ in this setting. We summarize:

Proposition 20. $(X, *(n))$ is separable over $(X, \cdot)$ for any $n$.

Suppose that $(X, *)=(\mathbb{R},+)$ and suppose that $x * y=a x+b y+c$ defines a linear groupoid. Then $x * y=(a x)+(b y+c)$ is separable over $(\mathbb{R},+)$. Furthermore, since $(\mathbb{R}, *(n))$ is also a linear groupoid, it is also separable in this case. Although somewhat similar to the previous case, it is not quite the same.

We shall say a binary system $(X, *)$ futuristic over $(X, \cdot)$ if $(\mathbb{R}, *(n))$ is separable over $(X, \cdot)$. The examples above are certain large classes. It may be of interest to determine separable and futuristic binary systems for certain types of binary systems. Notice that every binary system $(X, \cdot)$ is trivially separable over itself. Since we assume $(X, \cdot)$ is abelian, then $(x \cdot y) \cdot(y \cdot x)=x^{2} y^{2}$ and $x \cdot(n+1) y=x^{2^{n}} \cdot y^{2^{n}}$, so that it is also futuristic over itself. 
If $(X, \cdot)$ is the left zero semigroup and if $(X, *)$ is separable over $(X, \cdot)$, then $x * y=f(x) \cdot g(y)=f(x)$, i.e., $(X ; *, f)$ is a leftoid. Conversely, if $(X ; *, f)$ is a leftoid, then $x * y=f(x)=f(x) \cdot y$ for example, i.e., it is separable over the left zero semigroup.

$\S$ 13. Hopefully this short description of various perspectives has been sufficiently persuasive in providing evidence that the semigroups ( $\operatorname{Bin}(X), \square)$ are themselves of great interest and that they may be helpful in providing tools, questions and opportunities for applications in other areas of study.

Given what is known about iterated function systems and concepts like chaos, the butterfly effect, etc. associated with these and their orbits, the notion of attractors, both strange and otherwise, and its importance in the physical sciences it may be noted that any subsemigroup of $(\operatorname{Bin}(X), \square)$ is naturally an orbit, with the concepts like chaos, the butterfly effect, etc. naturally built in. Thus, e.g., the fact that strong (i.e., $x * y=y * x$ implies $x=y$ ) groupoids defined on a base set $X$ form a subsemigroup of $(\operatorname{Bin}(X), \square)$ indicates that one may use these to advantage in modeling physical systems should the opportunity arise.

The fact that different properties or axioms generate interesting substructures of $(\operatorname{Bin}(X), \square)$ for arbitrary sets $X$ may also serve as a classification level of these properties of axioms. One may think of a classification level somewhat along these lines: (i) level A: no special properties in general; (ii) level B: groupoids at this level for any set $X$ generate a subsemigroup; (iii) level C: groupoids at this level for any set $X$ generate a left ideal; (iv) level $\mathrm{C}^{\prime}$ : groupoids at this level for any set $X$ generate a right ideal; (v) level D: groupoids at this level for any set $X$ generate a two-sided ideal; (vi) level E: groupoids at this level for any set $X$ generate a subgroup (of units) of $(\operatorname{Bin}(X)$, $\square)$. Other distinctions can also be made. In the previous sections we have given many examples of properties at various levels.

For those taking the view of universal algebra or category theory, we note that our viewpoint is somewhat complementary in that we are not overly concerned with constructions such as direct products, sums, algebra types etc.. Our main construction is a composition of groupoids defined on a fixed set $X$. It is certainly possible to combine this construction with other notions such as those coming from category theory. To do so at this time seems highly premature in any case.

Again, the various perspectives provided above, along with the examples are meant to illustrate a number of concepts associated with the semigroups $(\operatorname{Bin}(X), \square)$ for sets $X$ which we believe are interesting themselves and which may be of use in applications also.

\section{References}

[1] R. L. O. Cignoli, I. M. L. D'ottaviano, and D. Mundici, Algebraic Ffoundations of Many-Valued Reasoning, Kluwer Academic Publishers, Dordrecht, 2000. 
[2] A. Dvurečenskij and S. Pulmannová, New Trends in Quantum Structures, Mathematics and its Applications, 516. Kluwer Academic Publishers, Dordrecht; Ister Science, Bratislava, 2000.

[3] J. S. Han, H. S. Kim, and J. Neggers, Strong and ordinary d-algebras, J. Multi-Valued Logic and Soft Computing (to appear).

[4] Y. Huang, BCI-algebra, Science Press, Beijing, 2006.

[5] J. Meng and Y. B. Jun, BCK-algebras, Kyung Moon Sa, Seoul, 1994.

[6] L. Nebeský, An algebraic characterization of geodetic graphs, Czechoslovak Math. J. 48(123) (1998), no. 4, 701-710.

7] _ A tree as a finite nonempty set with a binary operation, Math. Bohem. 125 (2000), no. 4, 455-458.

[8] _ Travel groupoids, Czechoslovak Math. J. 56(131) (2006), no. 2, 659-675.

[9] J. Neggers, A. Dvurečenskij, and H. S. Kim, On d-fuzzy functions in d-algebras, Found. Phys. 30 (2000), no. 10, 1807-1816.

[10] J. Neggers, Y. B. Jun, and H. S. Kim, On d-ideals in d-algebras, Math. Slovaca 49 (1999), no. 3, 243-251.

[11] J. Neggers and H. S. Kim, On d-algebras, Math. Slovaca 49 (1999), no. 1, 19-26.

HEE SIK KIM

Department of Mathematics

HANYANG UNIVERSITY

SEOUl 133-791, KoreA

E-mail address: heekim@hanyang.ac.kr

Joseph NEGGERS

Department of Mathematics

UNIVERSITY OF Alabama

TuscaloosA, AL, 35487-0350, U.S.A.

E-mail address: jneggers@as.ua.edu 